\title{
Spectrum-Aware Geographic Routing in Cognitive Vehicular Ad Hoc Network Using a Kalman Filter
}

\author{
Huma Ghafoor and Insoo Koo \\ School of Electrical Engineering, University of Ulsan (UOU), Muger-Dong San-29, Ulsan 680-749, Republic of Korea \\ Correspondence should be addressed to Insoo Koo; iskoo@ulsan.ac.kr
}

Received 5 August 2015; Revised 4 January 2016; Accepted 10 January 2016

Academic Editor: Jose R. Martinez-de Dios

Copyright ( $) 2016$ H. Ghafoor and I. Koo. This is an open access article distributed under the Creative Commons Attribution License, which permits unrestricted use, distribution, and reproduction in any medium, provided the original work is properly cited.

\begin{abstract}
We propose a position-based routing protocol for cognitive radio vehicular ad hoc networks (CR-VANETs) using a Kalman filter algorithm. The protocol first selects an idle channel from among all the channels available to a vehicle while moving on a straight road and then finds the best relay node to deliver the packet to the destination. The selection of a relay node is done by dividing the vehicular transmission range into five regions, and then the source/relay node selects the one that is in the region having a higher preference than other regions. Communication between two vehicles occurs only when both the vehicles are on the same channel. Delay may increase while sensing the channel and selecting the relay node. To reduce the delay, we use a Kalman filter to predict the future positions of all moving vehicles in the network. We consider vehicle-to-vehicle communication and compare our protocol for different probabilities of primary user being idle. Our proposed protocol shows improvement in both packet delivery ratio and end-to-end delay.
\end{abstract}

\section{Introduction}

The demand for road safety has increased due to the increase in the number of vehicles moving on the road. Communication among vehicles is becoming more important in order to deal with various applications while traveling. These applications include traffic safety to warn drivers about accidents and road conditions, traveling information like route information, hotel booking, and parking space booking and all other entertainment-related information. Therefore, cooperation among vehicles (for convenience, we use vehicle and node interchangeable in the rest of the paper) is essential for safe driving [1].

A vehicular ad hoc network (VANET) is a special class of mobile ad hoc network (MANET) that incorporates wireless technology in vehicles. It is the technology with which vehicles communicate with each other, with or without the help of roadside infrastructure, by moving in and out of the network. Communication with the aid of roadside infrastructure is called vehicle-to-infrastructure (V2I) communication. On the other hand, vehicle-to-vehicle (V2V) communication occurs only between vehicles, without considering roadside units [2].

The Federal Communications Commission (FCC) in the United States allocated $75 \mathrm{MHz}$ of spectrum at $5.9 \mathrm{GHz}$ for the exploitation of intelligent transportation system services in 1999. IEEE 802.11p/1609 is the standard for Dedicated Short Range Communication (DSRC), which is an amendment of IEEE 802.11. The European Union is also getting close to allocating $30 \mathrm{MHz}$ of spectrum at $5.9 \mathrm{GHz}$ for vehicular communications. DSRC channels are reserved for automobile communications only, but these have been found insufficient due to the increasing demands of vehicular applications. Therefore, the performance of vehicular networks may degrade when these channels are overloaded $[3,4]$.

For that reason, cognitive radio (CR) seems to be a technology to resolve this issue in VANETs. The performance of vehicular networks can be improved by letting vehicles use additional spectrum outside the DSRC band (e.g., TV bands) [5]. Vehicles are allowed to opportunistically sense the spectrum, provided they ensure the operation of the primary user (PU) is not affected. The implementation of 
a CR network in VANETs is different from generic CR networks due to the fact that, in CR-VANETs, two important factors must be considered: dynamic behavior of vehicles and their cooperation with each other.

Routing in VANETs has been considered a critical issue in both academia and industry for decades. Due to highspeed vehicles, sparse network conditions, and difficulty in tracking destination vehicles, existing routing protocols for MANETs are not suitable for VANETs. Therefore, in VANETs, it is difficult for vehicles to maintain a route between source and destination. A lot of work has been done on proposing routing protocols in both MANETs and VANETs. Some protocols have been proposed for CR-MANETs. These protocols cannot be directly applied to CR-VANETs for the above reasons. Very few protocols have been proposed for routing in CR-VANETs; the literature has been more focused on spectrum sensing and spectrum management for CRVANETs.

In this paper, a novel spectrum-aware geographic routing protocol is proposed for $\mathrm{V} 2 \mathrm{~V}$ communication in highway scenarios. The vehicles are moving on a straight road in a two-way direction. The road is divided into a number of segments of equal length. The vehicles are allowed to use any of the licensed TV channels when PU activity is not affected. Spectrum sensing is performed with the cooperation of the vehicles, so that each vehicle has the list of spectrum holes, which is updated among neighboring vehicles as an additional entry in a beacon message. Vehicles periodically update each other as to their current geographic positions, and the Kalman filter is used to predict their future positions. The relay is selected by dividing the vehicle's transmission range into five regions.

The remainder of the paper is organized as follows. Related work is presented in Section 2. In Section 3, we propose the spectrum-aware geographic routing protocol using a Kalman filter algorithm. Section 4 presents the performance results, and finally, Section 5 provides the concluding remarks.

\section{Related Work}

Routing in CR-VANETs is more challenging as compared to conventional routing protocols in VANETs. Geographic routing protocols for VANETs are divided into three main categories: nondelay tolerant network (non-DTN), delay tolerant network (DTN), and hybrid. Many studies have been conducted to propose protocols (listed a few: GPSR [6], VADD [7], and GeoDTN+Nav [8]) in these categories for efficient routing in VANETs. A detailed comparison of these protocols can be seen in our previous work [2]. These protocols aimed to provide end-to-end connectivity considering latency and packet delivery ratio as essential metrics. As the demand for multimedia applications increases, congestion in vehicular networks and spectrum scarcity also increase [9]. These existing routing protocols in the literature mainly focus on successful delivery of packet without considering the overburdened routes and scarcity of spectrum [10].

To the best of our knowledge, research in CR-VANETs is very limited. CoRoute [11] is one of the known routing protocols in the area of CR-VANETs for city scenarios. It is an any-path routing protocol that differs from conventional $\mathrm{CR}$ approaches in that it uses the unlicensed band and utilizes multiple channels by allowing vehicles to coexist with the PU on the same channel. CoRoute uses estimated transmission time (ETT) as a route metric to estimate the transmission time for a node two hops away from the querying node. It achieved $100 \%$ improvement in throughput by considering the destination node two hops away from the source node. CoRoute does not ensure the same performance achievement for long routes, where the destination is far from the source node.

Liu et al. [12] proposed another routing algorithm for CR-VANETs on a freeway map. In this algorithm, they first calculated the expected link duration and then selected the route that has the longest path duration. They assumed that each vehicle can detect the spectrum accurately and that they have geographic information about neighbors from a global positioning system (GPS). They did not consider cooperative spectrum sensing among vehicles. This algorithm calculated relative distance, relative speed, and path duration. Their results showed robustness in the network by considering the ideal quality of the channel.

Spectrum-Aware Beaconless (SABE) geographic routing [13] is a protocol proposed for CR-VANETs in which TV bands are shared dynamically among cognitive radio-enabled vehicles. This protocol uses one or more "CR information polls" (i.e., base stations) to obtain a list of TV channels that are free from PU activity. A relay node is chosen with channels, transmission powers, and transmission rates jointly by cognitive radio-enabled vehicles. The process does not broadcast beacons. The information is acquired by overhearing control packets.

All of these existing routing protocols for CR-VANETs have some restrictions on the selection of both channel and relay node. They make assumptions to improve the success rates of their protocols. To reduce the delay in VANET environment, they consider fixed units. Due to the dynamic nature of both channels and vehicles, the network topology in CR-VANETs changes very fast. Therefore, an exact location of vehicles is difficult to determine in such rapid changing environment. Consequently, there is a need for an efficient routing protocol in CR-VANETs. This work proposes a routing protocol in CR-VANETs which selects the best relay node by combining cognitive capability and prediction technique in order to overcome the problems of spectrum scarcity and high latency in VANETs. Moreover, the protocol is implemented for long-distance paths between source and destination without considering roadside units. This is a multihop multichannel cognitive routing scheme in which spectrum sensing is done by cooperation of the neighboring vehicles. Our goal is for communication to be entirely V2V in highway scenario, without using any fixed unit.

\section{Proposed Protocol}

We propose a position-based routing protocol for cognitive vehicular ad hoc networks in a highway scenario. The 


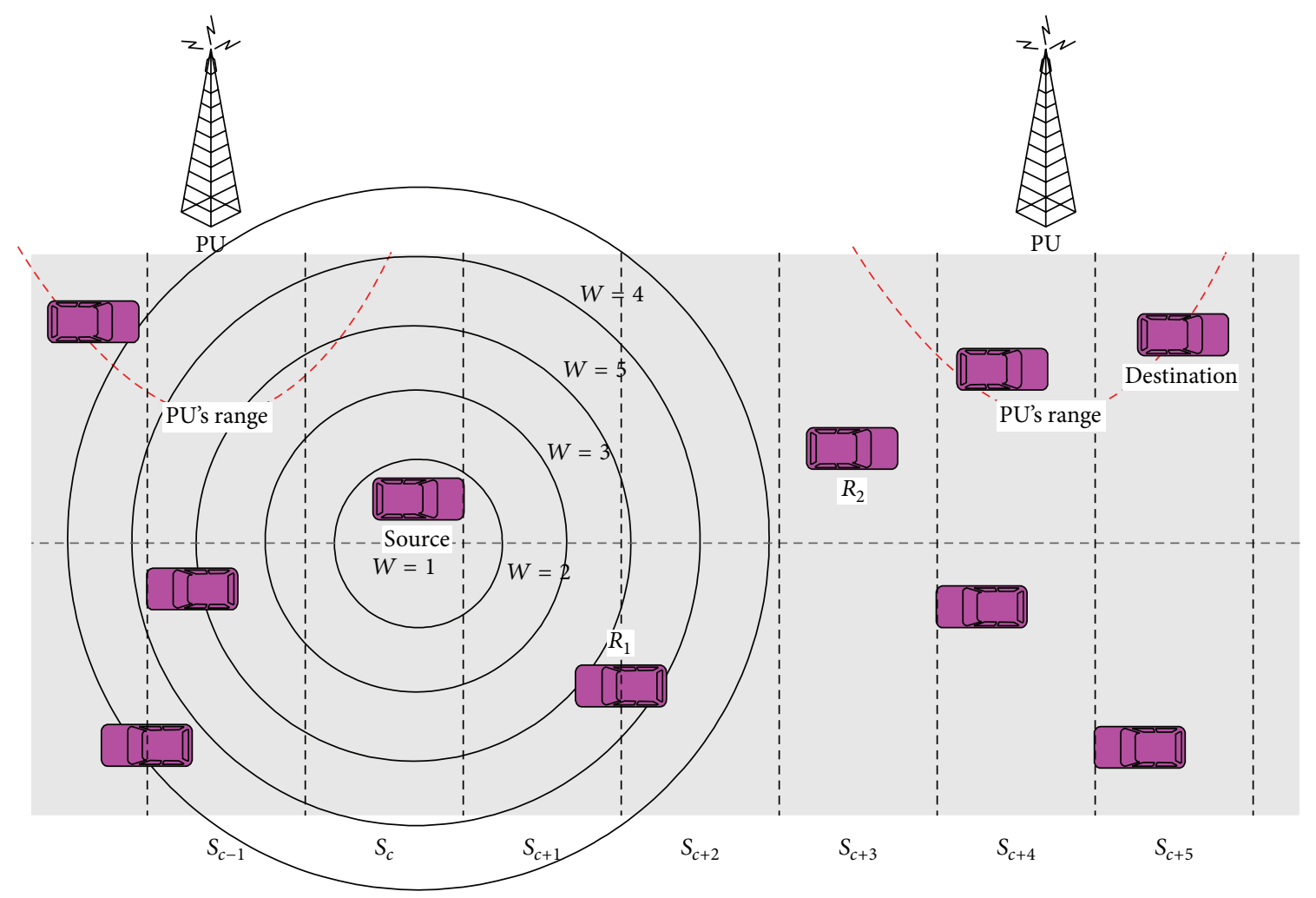

FIGURE 1: An example illustrating channel and relay selection in a highway scenario.

purpose of using cognitive principles in a vehicular ad hoc network is to increase the spectrum opportunities for V2V communication. Like existing vehicular routing protocols in the literature, this protocol also predicts the future positions of vehicles by using a Kalman filter. The Kalman filter helps to reduce end-to-end delay while making routing more efficient since delay is a critical issue in all routing algorithms.

The proposed protocol is a spectrum-aware geographic routing protocol. As the name implies, it first senses the spectrum with the cooperation of vehicles moving on a straight road and then selects the best relay node closest to the destination which has the same idle channel as the source/relay node. The basic goal is to establish a stable routing path between source and destination. It is cognitive $\mathrm{V} 2 \mathrm{~V}$ communication in which all the nodes are moving vehicles. Each vehicle is assumed to be equipped with a GPS receiver to get its current position. The highway road is assumed to be divided into segments of equal length, and digital maps are assumed to be installed in each vehicle, so each vehicle is aware of information about the segment it is moving on. The highway roads generally have multiple lanes but we assume two separate lanes for vehicles moving in opposite directions (West-East and East-West) as shown in Figure 1. We also assume that the TV band is divided into $M$ channels, and each channel can be utilized by a licensed PU. Spectrum sensing is done by the energy detector. We initially consider one-way direction of the vehicles and then extend this work to find network connectivity when vehicles are moving in two-way direction.
Considering vehicles moving in a highway scenario, we divide the straight road into $S$ segments of equal length, $l$. The proposed protocol is twofold. First, cooperative spectrum sensing is performed by merging the sensing information collected by each vehicle individually, and then the best route is selected on the basis of each node's transmission range weight factor from among the entire detected idle channels. In the following section, we discuss these two parts.

3.1. Spectrum Sensing. We consider cognitive V2V communication in which both source and destination are moving vehicles. The radio channel for $\mathrm{V} 2 \mathrm{~V}$ communication is affected by three factors: path loss, small-scale fading, and large-scale fading $[14,15]$. These factors have different impact on system performance depending on the line-of-sight (LOS) path between transmitter and receiver. In highway scenarios, the LOS path exists between communicating vehicles if their communication range is short and there is no obstacle in the path. However, when the distance between two communicating vehicles is large and traffic load is heavy, a non-LOS (NLOS) component might occur. Usually, the path loss in the former case is modeled by two-ray path loss model and the Rician distribution is used to model small-scale fading. In the latter case, the log-distance path loss model is more suitable for NLOS cases and the Rayleigh distribution is used to model small-scale fading. Log-normal distribution is used to model large-scale fading in both cases [16].

In this paper, we consider the Nakagami distribution to model the fading of PU activity as the distribution describes 
statistical characteristics of both small-scale and large-scale fading $[14,15]$. Since the proposed scheme is for cognitive $\mathrm{V} 2 \mathrm{~V}$ communication, spectrum sensing is the first step to approach a free channel and starts the communication. In cognitive V2V communication, fading effect may degrade the sensing performance. Therefore, in the paper, we examine the effect of the Nakagami fading of PU activity on the performance of energy detector-based spectrum sensing scheme by merging the local sensing results of each vehicle with its neighbors to reduce fading impact on PU sensing activity since individual vehicle cannot reliably detect the presence/absence of PU due to fading.

The communication model for spectrum sensing is based on the IEEE $802.11 \mathrm{p} / 1609.4$ protocol [17] in which control or management information and data transmission switch between a common control channel $(\mathrm{CCH})$ and a service channel $(\mathrm{SCH})$ at regular intervals, respectively. Both channels have the same interval of $50 \mathrm{~ms}$. In this paper, we consider the same modification in the communication model as suggested by Felice et al. [18]. A SCH slot is fixed for sensing activity after every $150 \mathrm{~ms}$. The interval of sensing activity is equal to SCH slot, that is, $50 \mathrm{~ms}$. Each vehicle can select any of the $M$ channels and periodically senses the spectrum by using the energy detector [19].

We consider the cooperative spectrum sensing [18] in order to obtain the list of sensing results (LSR) of each vehicle moving within range of the spectrum horizon size, $h$. Spectrum horizon size, $h$, is the range of highway segments up to which each vehicle keeps record of spectrum availability in its list. The results from the sensing activity are stored in a spectrum availability table, which has the following contents for each vehicle:

$$
\left\langle S, f, E_{S, f}, n_{S, f}\right\rangle,
$$

where $S$ represents the segment where the vehicle is moving, $f$ is one of the $M$ channels, $E_{S, f}$ is the mean value of the power of the signal at channel $f$ and segment $S_{k}(k$ can be $c, c+h$ or $c-h$, where $c$ represents current state), and $n_{S, f}$ is the number of samples at channel $f$ and segment $S_{k}$.

Each vehicle keeps a record of the information in its spectrum table for segments to horizon size, $h$. As vehicles are moving, their segments will change continuously. Therefore, vehicles update their horizon size $h$ for the next segment it is moving on and remove entries of the previous segments automatically. Thus, any information outside the range of horizon size $h$ of each vehicle will automatically be deleted from the table. Vehicles periodically update their spectrum table at a fixed interval. If vehicle $R_{1}$ moving in segment $S_{c+1}$ receives a message from vehicle $R_{2}$, which is moving in the next segment and is within communication range of $R_{1}$, the table of $R_{1}$ is updated by the following merging rule [18]:

$$
E_{S, f}^{R_{1}}=(1-\alpha) E_{S, f}^{R_{1}}+\alpha E_{S, f}^{R_{2}},
$$

where $\alpha$ is the ratio of number of sensing samples in vehicle $R_{2}$ to total number of sensing samples between two vehicles as defined by

$$
\alpha=\frac{n_{S, f}^{R_{2}}}{n_{S, f}^{R_{1}}+n_{S, f}^{R_{2}}} .
$$

Each vehicle then detects ON/OFF PU activity for the given horizon size $h$ by using binary hypothesis model which is defined as

$$
H(S, f)_{R_{1}}= \begin{cases}0 & \text { if } E_{S, f}^{R_{1}} \leq \rho \\ 1 & \text { if } E_{S, f}^{R_{1}}>\rho .\end{cases}
$$

On the basis of this information, each vehicle now has LSR for all the channels in its path for the horizon size, $h$. Therefore, in this way, each vehicle knows the availability of channel in the next segment in advance. As vehicles are moving in two-way direction we consider LSR for the previous, current, and future segments (for one-way scenario LSR depends on current and future segments only): that is,

$$
\operatorname{LSR}=\left\{H\left(S_{i}, f\right)\right\}
$$

where $S_{i}=\left[S_{c-h}, S_{c}, S_{c+h}\right]$.

Now, this list of holes is used by the source/relay node to select the best relay node for establishing a routing path to the destination. This selection is done by choosing the common idle channel from the list when two nodes intend to communicate with each other in order to form the path between source and destination. The following section explains how routing is done in $\mathrm{V} 2 \mathrm{~V}$ communication using the LSR.

3.2. Relay Selection. In this subsection, we explain how the proposed routing protocol works by using the LSR obtained in the previous subsection. We divide the vehicular range of each node into five regions $(r / 5,2 r / 5,3 r / 5,4 r / 5$, and $r)$ [20], where $r$ is the radius of the circle. Each region is assigned a weight factor, $W$, in the following manner. The maximum value $W=5$ is assigned to the vehicles moving between $3 r / 5$ and $4 r / 5$. $W=4$ is assigned to vehicles moving between $4 r / 5$ and $r, W=3$, to vehicles moving between $2 r / 5$ and $3 r / 5, W=2$, to those moving between $r / 5$ and $2 r / 5$, and $W=1$, for vehicles moving within the range $r / 5$. The reason for assigning the maximum value $W=5$ in that region is to minimize the number of dropped packets by giving a lower preference to nodes moving near the boundary of the circle, because there are more chances for those vehicles to move out of communication range. In geographical routing such as GPSR [6] (a benchmark scheme), a node is selected as a relay node which is closest to the destination, that is, selecting the furthest node from the source node. Our scheme is jointly handling two critical issues: selection of channel as well as relay node. We intend to increase the packet delivery ratio by decreasing the packet delay and overhead. Therefore, if we assign $W=5$ near the boundary of the circle, vehicles may move out of communication range quickly due to their 
varying speed. By knowing the future positions of vehicles using the Kalman filter, the querying node may know that a relay node near the boundary of circle moving with higher speed then the querying node will be out of communication range shortly in the future positions. Also, if we assign $W=$ 5 closer to the source node, the number of hops will be increased which increases the overhead. For that reason, delay will also be increased due to selecting the channel for large number of hops. Therefore, the region between $3 r / 5$ and $4 r / 5$ is safer than others such that there are fewer chances for the source/relay node to create multiple hops within its transmission range.

To initiate routing, each node first broadcasts a beacon message to all its neighboring nodes. We assume that all nodes are periodically sending beacon messages to update neighbors with their current status. Also, we assume that the source node can get the current position of the destination node using a reactive location service algorithm [21]. The beacon message includes the following entities: the vehicle's ID, position, speed, and LSR:

$$
\langle\text { ID, position, speed, LSR〉, }
$$

where position coordinates $(x, y)$ are calculated using the GPS and speed is calculated using the equation $\sqrt{\left(x_{2}-x_{1}\right)^{2}+\left(y_{2}-y_{1}\right)^{2}} /[(t-1)-t] .\left(x_{1}, y_{1}\right)$ is the previous position of the vehicle at time $t-1$, and $\left(x_{2}, y_{2}\right)$ is the current position at given time $t$. The future position of all moving vehicles is predicted by the Kalman filter. We will discuss the Kalman filter in the next subsection. Each node updates its table by receiving the beacon messages from neighboring nodes. Then the source node selects the best relay node among all the neighboring nodes in its transmission range using the following strategy.

Let us assume some vehicles are moving on the highway, as shown in Figure 1. We assume that transmission range of the PU may affect several segments. By considering the example scenario in Figure 1, we can better understand how the source node selects the relay node to communicate with the destination node. The source node, which is in the current segment $S_{c}$ in the region $W=1$, wants to communicate with a destination node outside its communication range, that is, in segment $S_{c+5}$. After sending the beacon message to all the nodes within its transmission range, the source node calculates $W$ for all its neighbors and then chooses the relay node that has the following two factors: (1) relay node and source node are on the same channel, which is selected from the LSR (here, it is noteworthy that in the paper we adopt a random selection when there is more than one same channel between two communicating vehicles. An efficient allocation scheme may be considered as a future work), and (2) the relay node is in the region $W=5$ and is closer to the destination than other nodes. In the region $W=5$, there is only one car $R_{1}$ moving in the opposite direction of the destination. Therefore, $R_{1}$ is selected as the first relay node. Then, $R_{1}$ repeats the same steps to find another relay node. The same procedure is repeated until the message reaches the destination node. If there is no node in range $W=5$, then preference will be given to other values of $W$ accordingly.
Using this approach, there are more chances for a packet to reach the destination safely without the risk of a large number of dropped packets, but performance may be affected due to selection of the same idle channel. The flowchart for relay selection mechanism is represented in Figure 2.

3.2.1. Kalman Filter Model. To make routing efficient by achieving a reasonable end-to-end delay, we use a Kalman filter in our proposed routing protocol to predict future positions of all vehicles on the highway. As we assume that vehicles are equipped with GPS, it provides an estimation of position within a few meters. Therefore, there must be some errors due to environmental factors that can degrade network performance. By using the Kalman filter algorithm, an estimation of the state from a series of noisy measurements is calculated $[22,23]$.

The Kalman filter has two vectors [23] (state vector and measurement vector) and it consists of two equations: a process equation and a measurement equation. The state vector consists of position $P_{t}$ and speed $v_{t}$ of a vehicle: that is, $u_{t}=\left[\begin{array}{l}P_{t} \\ v_{t}\end{array}\right]$. The process equation predicts the future value of the vehicles at time $t+1$, depending on the given time $t$; and the measurement equation, which is also called an observation equation, gives the observation value from the GPS. The process equation and measurement equation are defined by (7) and (8), respectively:

$$
\begin{gathered}
u_{t+1}=A u_{t}+\varepsilon_{u}, \\
z_{t}=H u_{t}+\varepsilon_{z},
\end{gathered}
$$

where $A$ and $H$ are state transition and measurement matrices, respectively, $\varepsilon_{u}$ is process noise, and $\varepsilon_{z}$ is measurement noise. Both noises have a normal distribution with zero mean and a covariance matrix of $Q$ and $R$, respectively.

The algorithm provides a set of mathematical equations to predict the unknown state on the basis of measurement values. A more in-depth description of the Kalman filter can be found in [24]. The following equations show how the Kalman filter measures the predicted and new values of moving vehicles. For this purpose, the algorithm is further divided into two steps: a prediction step and an update step. As the name implies, the prediction step predicts the state and covariance at time $t+1$, whereas the update step measurement is done by a combination of predicted state and observation value. The predicted values of state and covariance are defined as

$$
\begin{aligned}
& \widehat{u}_{t+1}=A u_{t}, \\
& P_{t+1}=A P_{t} A^{T}+Q .
\end{aligned}
$$

And the new estimated values of each vehicle are defined as

$$
\begin{aligned}
& \widehat{u}_{t+1 \mid t+1}=\widehat{u}_{t+1 \mid t}+K\left[z_{t+1}-H \widehat{u}_{t+1 \mid t}\right], \\
& P_{t+1 \mid t+1}=P_{t+1 \mid t}-K\left(R+H P_{t+1 \mid t} H^{T}\right) K^{T},
\end{aligned}
$$

where $K$ is the Kalman gain calculated from

$$
K=P_{t+1 \mid t} H^{T}\left[R+H P_{t+1 \mid t} H^{T}\right]^{-1} .
$$




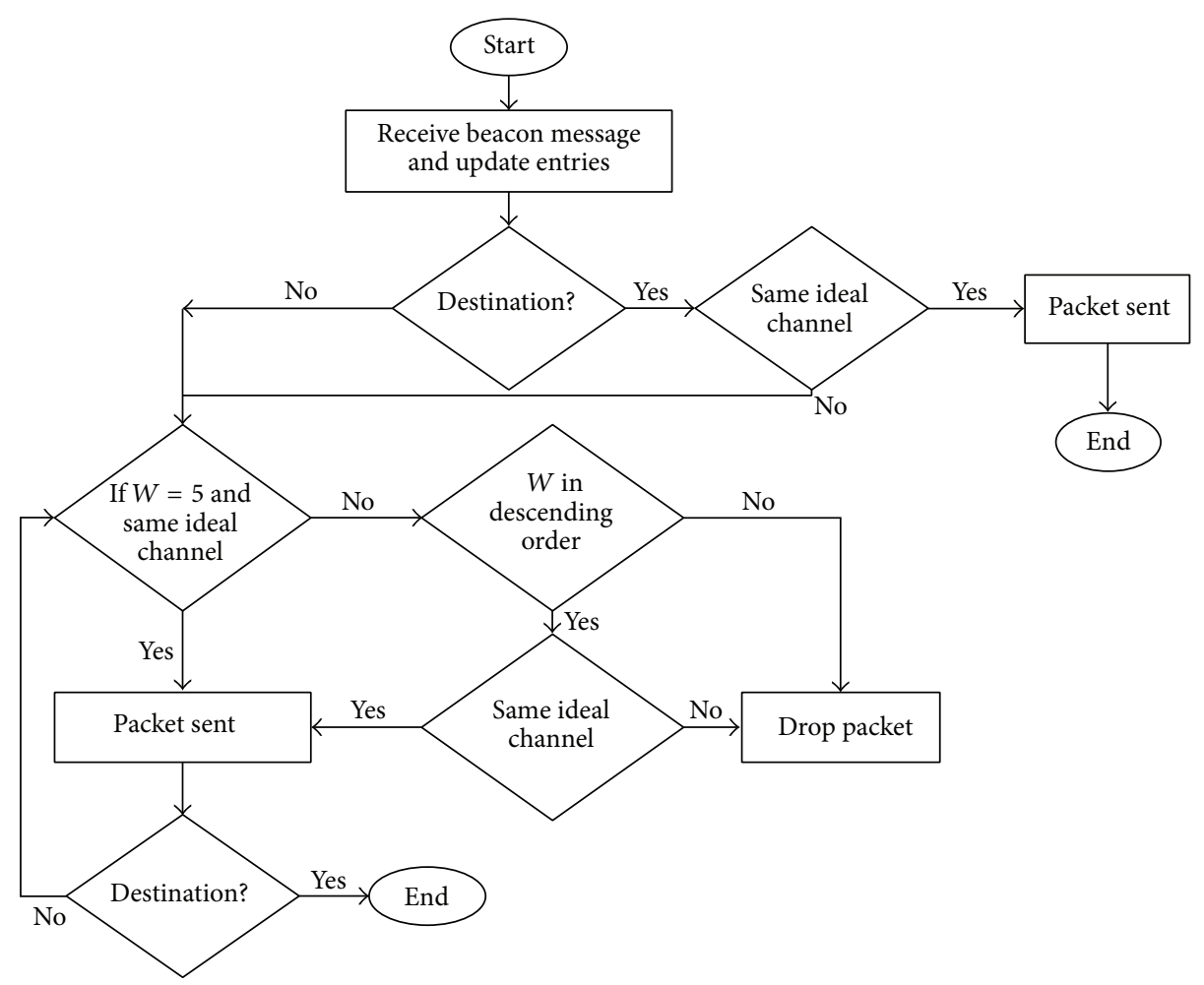

FIgURE 2: A flowchart representing relay selection mechanism.

Therefore, we can estimate the future values of position and velocity of vehicles by using the Kalman filter algorithm.

\section{Performance Evaluation}

We evaluate the performance of our proposed protocol in MATLAB using the example highway scenario as shown in Figure 1 . We divide the highway into 15 equal segments, each a $100 \mathrm{~m}$ length. The spectrum band is divided into $M=5$ channels and each channel can be occupied by a licensed PU. Nakagami propagation model is considered to model the fading impact on PU activity. Horizon size, $h=1$. In this example scenario, vehicles are moving at various speeds from $10 \mathrm{~m} / \mathrm{s}$ to $40 \mathrm{~m} / \mathrm{s}$. The number of relay nodes varies from 10 to 30 , each having a transmission range, $r=200 \mathrm{~m}$. Our simulation results are the average of 15 runs.

For simplicity, we denote our proposed protocol as the Kalman filter-based Cog-VANET. A comparison is made between the Kalman filter-based Cog-VANET and the classic Cog-VANET using different probabilities of the PU being idle. Classic Cog-VANET is a combination of two schemes proposed by Felice et al. [18] and Dhurandher et al. [20]. We simulate these two schemes in MATLAB and name them as Classic Cog-VANET. We evaluate our protocol for both one-way and two-way directions of the vehicles to find network connectivity. Three metrics are used to evaluate the performance of our proposed protocol:

(a) packet delivery ratio; (b) end-to-end delay;

(c) routing overhead.

First, we evaluate the packet delivery ratio of the Kalman filter-based Cog-VANET and the classic Cog-VANET for different idle probabilities of PU for both one-way and twoway highway scenarios. We take the number of relay nodes as an independent variable and we select only those nodes as relay nodes that are moving between source and destination. The nodes moving away from any current positions of source and destination have not been considered in this work. Packet delivery ratio is defined as the ratio of number of packets delivered to the destination to number generated by the source node. Figure 3 shows that the delivery ratio increases with an increase in the number of vehicles. By predicting the future positions of vehicles, we achieve $82 \%$ packet delivery for one-way highway scenario and $87 \%$ for two-way highway scenario when there are more vehicles on the road as shown in Figures 3(a) and 3(d), respectively. Moreover, the Kalman filter-based Cog-VANET outperforms the classic Cog-VANET in both scenarios, even when there are fewer vehicles because predicting the future positions helps the querying node to find the best relay node that makes stable routing path. Figures 3(b)-3(c) and Figures 3(e)3(f) show packet delivery ratios with different probabilities of PU $P\left(H_{0}\right)$ for the Kalman filter-based Cog-VANET and the classic Cog-VANET for both one-way and two-way scenarios, respectively. As the probability that the $\mathrm{PU}$ remains idle decreases, the performance of the delivery ratio decreases. 


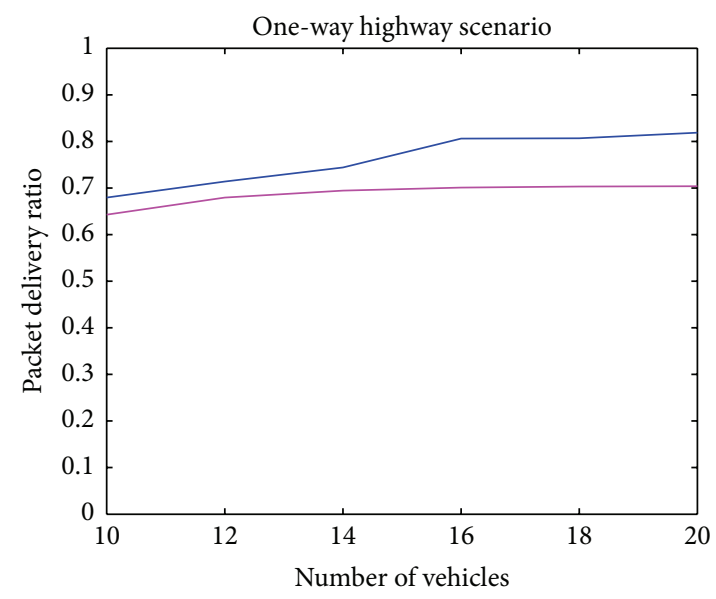

- Kalman filter-based Cog-VANET with $P\left(H_{0}\right)=1$ _ Classic Cog-VANET with $P\left(H_{0}\right)=1$

(a)

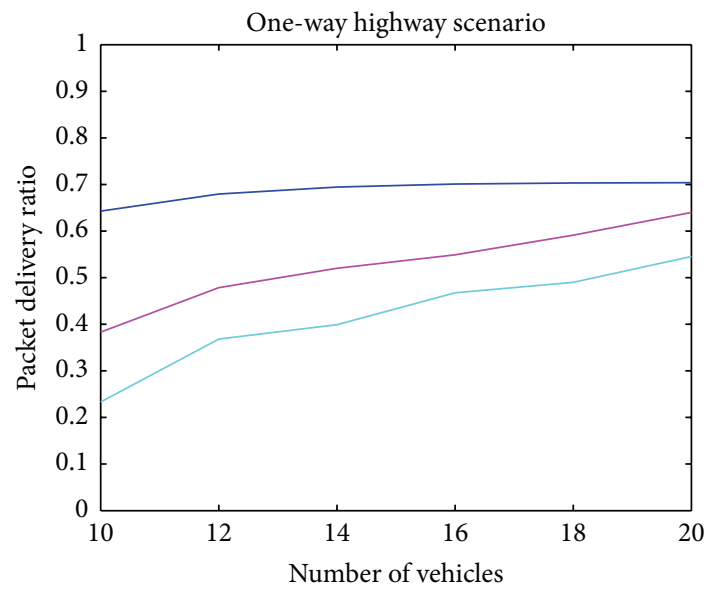

- Classic Cog-VANET with $P\left(H_{0}\right)=1$

- Classic Cog-VANET with $P\left(H_{0}\right)=0.8$

— Classic Cog-VANET with $P\left(H_{0}\right)=0.6$

(c)

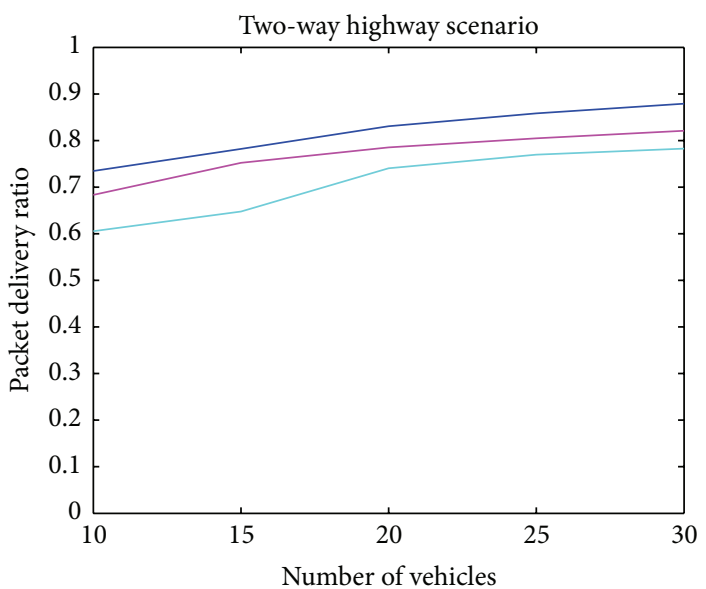

- Kalman filter-based Cog-VANET with $P\left(H_{0}\right)=1$

- Kalman filter-based Cog-VANET with $P\left(H_{0}\right)=0.8$

- Kalman filter-based Cog-VANET with $P\left(H_{0}\right)=0.6$

(e)

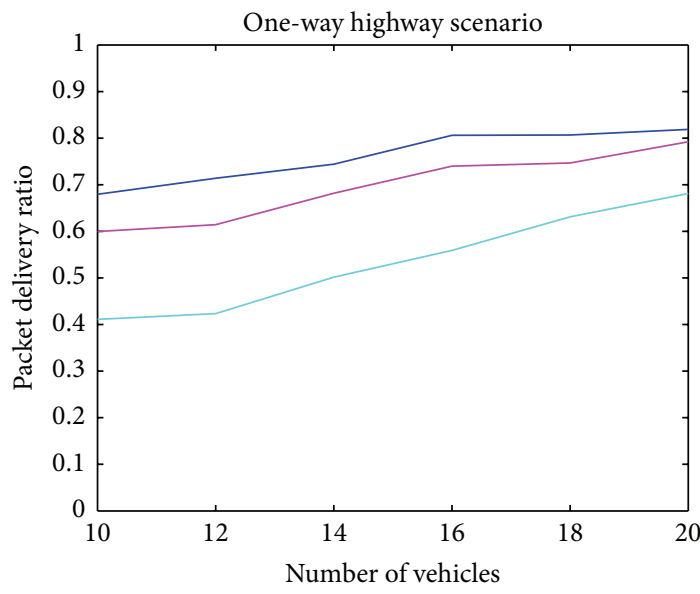

- Kalman filter-based Cog-VANET with $P\left(H_{0}\right)=1$

- Kalman filter-based Cog-VANET with $P\left(H_{0}\right)=0.8$

Kalman filter-based Cog-VANET with $P\left(H_{0}\right)=0.6$

(b)

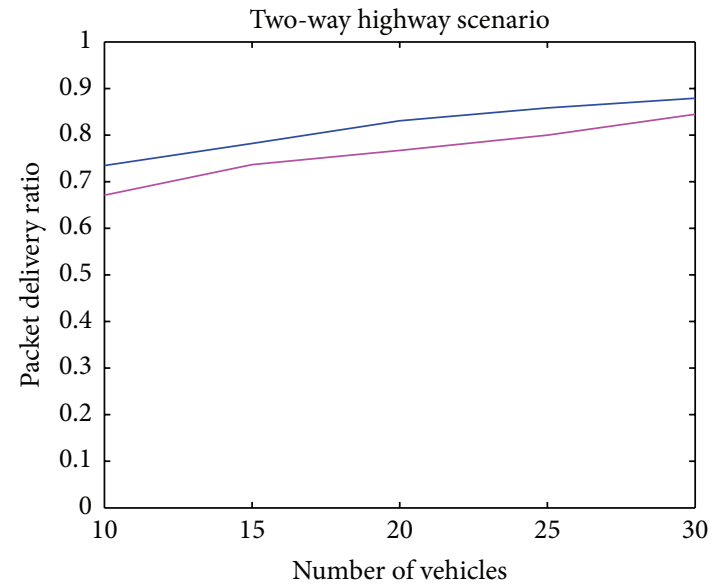

- Kalman filter-based Cog-VANET with $P\left(H_{0}\right)=1$

- Classic Cog-VANET with $P\left(H_{0}\right)=1$

(d)

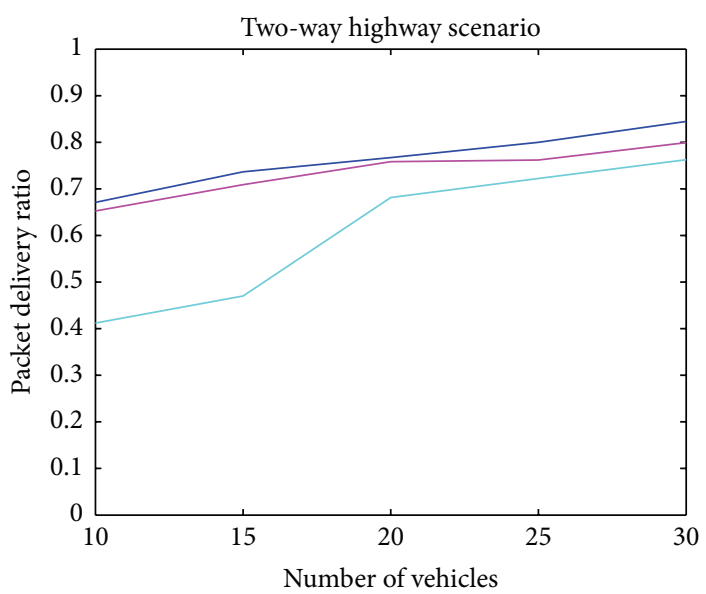

- Classic Cog-VANET with $P\left(H_{0}\right)=1$

— Classic Cog-VANET with $P\left(H_{0}\right)=0.8$

— Classic Cog-VANET with $P\left(H_{0}\right)=0.6$

(f)

Figure 3: Continued. 


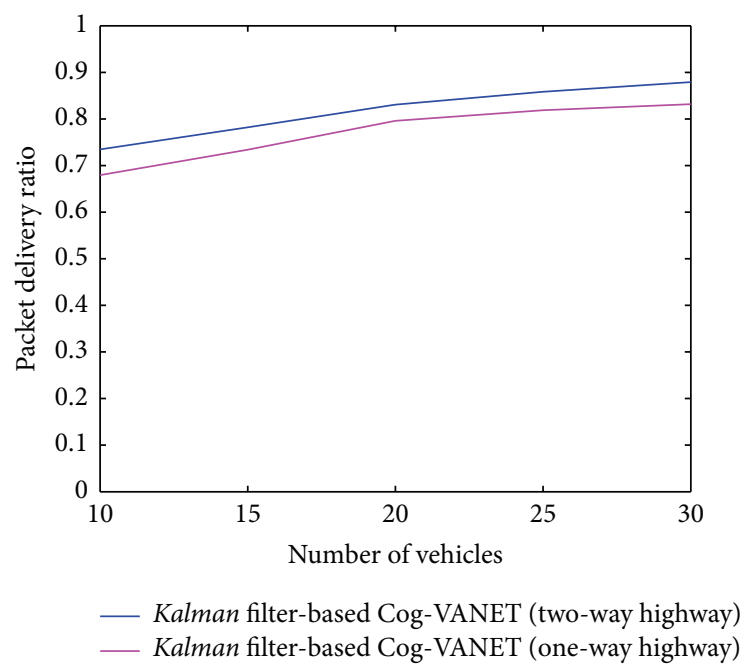

(g)

FIGURE 3: Performance comparison between the Kalman filter-based Cog-VANET and the classic Cog-VANET for one-way and two-way highway scenarios in terms of packet delivery ratio for different idle probabilities of the PU.

The reason for decrease in delivery ratio is facing difficulty in finding common free channel when the idle probability of PU decreases. It means that the channel is being occupied by the licensed user for long duration of time. Hence, vehicles do not have common idle channel to communicate with each other when probability of PU $P\left(H_{0}\right)$ decreases. This increases the network fragility which degrades overall network performance. It is also noteworthy from Figures 3 (b) and 3(c) that when there is less number of nodes in the network, the delivery ratio decreases to $41 \%$ in Kalman filter-based Cog-VANET and 23\% in classic Cog-VANET for one-way highway scenario. However, for two-way scenario when there is less number of nodes as shown in Figures 3(e) and $3(\mathrm{f})$, the delivery ratio is comparatively better because of having knowledge of both previous and future segments. Hence, the overall performance is found to be degraded when the network is sparse due to not finding an idle channel as well as best relay node to make communication possible. Nevertheless, it is clearly depicted from Figure 3 that the Kalman filter-based Cog-VANET outperforms the classic Cog-VANET in all scenarios. Therefore, we conclude that the availability of the channel, prediction of vehicles' next positions, and the connectivity of the network are important factors for efficient performance of the network. Figure $3(\mathrm{~g})$ shows the comparison between two different highway scenarios. It can be clearly depicted from figure that vehicles moving in opposite directions in two-way scenario provide stable paths between source and destination which increases packet delivery ratio and therefore increases the connectivity of the network. Also, due to the fact that LSR has information of both previous and future segments in two-way scenario, it performs better than one-way scenario.

Figure 4 shows the performance of end-to-end delay for both the Kalman filter-based Cog-VANET and the classic Cog-VANET considering both highway scenarios. End-toend delay is defined as the difference between the start time and the end time of a packet going from source to destination. As the number of relay nodes increases, network connectivity improves; therefore, end-to-end delay decreases. The reason that the delay for the Kalman filter-based Cog-VANET shows better performance is due to prediction of future positions of all the vehicles. In the classic case, the source node has to wait for a reply to the beacon message for every entry in the routing table. However, using the Kalman filter, one can predict the future positions, which save route time and reduce the number of beacon messages that need to be sent to the destination node. Another factor that affects the performance of end-to-end delay is the selection of an idle channel. We can also see from Figure 4 that when considering the case of vehicles moving in the opposite lane, both classic CogVANET and Kalman filter-based Cog-VANET perform better than one-way scenario. The reason for the less delay in twoway scenario is due to connectivity and exchange of channel information of previous segments as well. By considering the vehicles moving in opposite lanes, the LSR has more information about the state of channels at different locations. Consequently, selecting an idle channel in two-way scenario consumes less time than one-way scenario. Therefore, delay is longer in case of classic Cog-VANET one-way scenario because nodes consume more time in finding next positions of their neighboring nodes as well as in reselecting an idle channel.

Figure 5 shows the impact of routing overhead when number of nodes in the network is increased. The routing overhead is defined as the number of beacon messages needed to deliver a packet to the destination. The number of beacon messages increases with the increasing number of nodes. By predicting the future positions, vehicles do not need to search for next locations; therefore, the number of beacon messages for Kalman filter-based Cog-VANET in both scenarios is always less than classic Cog-VANET. We also observe that the routing overhead for two-way highway 


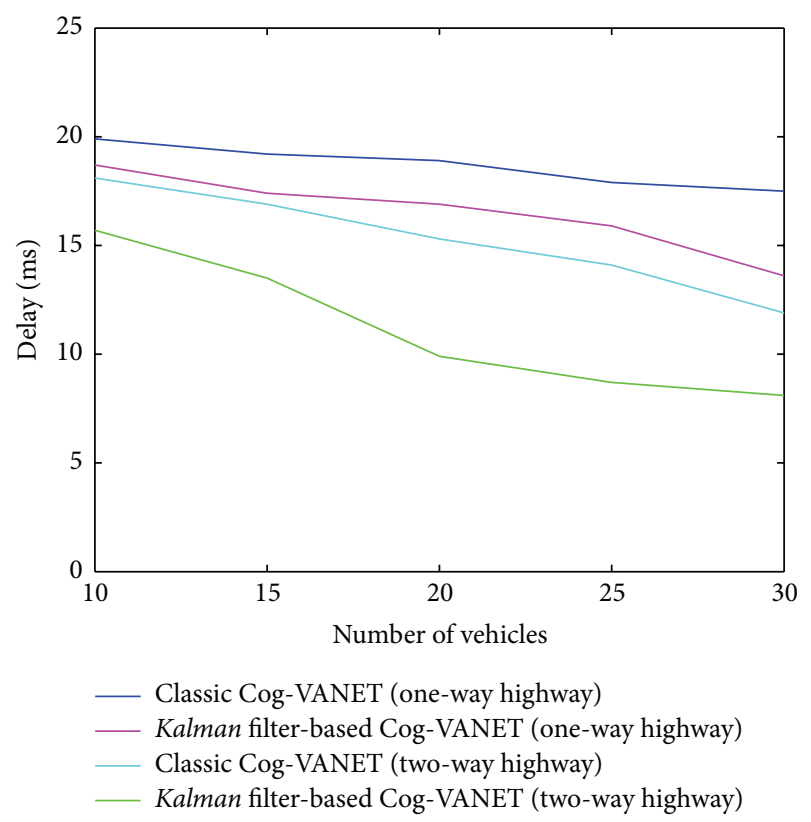

FIgURE 4: Performance comparison between the Kalman filterbased Cog-VANET and the classic Cog-VANET for one-way and two-way highway scenarios in terms of end-to-end delay.

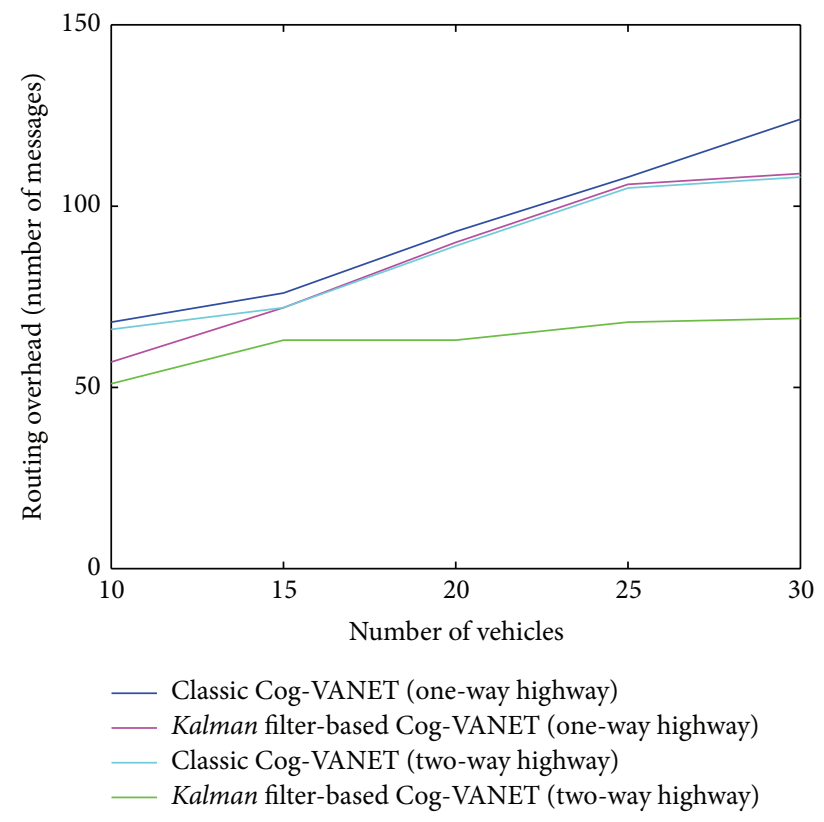

FIGURE 5: Performance comparison between the Kalman filterbased Cog-VANET and the classic Cog-VANET for one-way and two-way highway scenarios in terms of routing overhead.

scenario for the same number of nodes in each protocol is less than the routing overhead for one-way highway scenario of their corresponding protocol. Hence, the Kalman filter increases the overall performance of the network by reducing routing overhead as well as end-to-end delay.

The impact of idle probability of PU for both metrics (end-to-end delay and routing overhead) provides results in the same pattern for each scenario as shown in Figure 3; that is, with an increase in probability we experience a decrease in both end-to-end delay and routing overhead. A complete analysis of our simulation results show that as packet delivery ratio increases, end-to-end delay and routing overhead for both one-way and two-way highway scenarios decrease accordingly. Therefore, for the sake of space limitation we omit the results that show the effect of different idle probabilities of PU on end-to-end delay and routing overhead.

\section{Conclusion}

In this paper, we have proposed a novel routing protocol for cognitive vehicular ad hoc networks for highway scenarios. The combination of both channel selection and relay selection in vehicle-to-vehicle communication without considering any roadside units for long-distance paths between source and destination makes this protocol unique from all the existing protocols of cognitive VANET in the literature. This is a position-based routing protocol in which the Kalman filter is used to predict the future positions of the vehicles. With the use of the Kalman filter algorithm, our protocol shows better performance for packet delivery ratio and endto-end delay. In the future, we will extend this work to city scenarios and also for different channel propagation models in both highway and city scenarios.

\section{Conflict of Interests}

The authors declare that there is no conflict of interests regarding the publication of this paper.

\section{Acknowledgment}

This work was supported by the National Research Foundation (NRF) of Korea funded by the MEST (nos. NRF2013R1A2A2A05004535 and NRF-2015R1A2A1A15053452).

\section{References}

[1] K. D. Singh, P. Rawat, and J.-M. Bonnin, "Cognitive radio for vehicular ad hoc networks (CR-VANETs): approaches and challenges," EURASIP Journal on Wireless Communications and Networking, vol. 2014, article 49, 2014.

[2] H. Ghafoor, I. Koo, and N.-U.-D. Gohar, "Neighboring and connectivity-aware routing in VANETs," The Scientific World Journal, vol. 2014, Article ID 789247, 10 pages, 2014.

[3] D. Jiang and L. Delgrossi, "IEEE 802.11p: towards an international standard for wireless access in vehicular environments," in Proceedings of the IEEE Vehicular Technology Conference (VTC '08), pp. 2036-2040, IEEE, Singapore, May 2008.

[4] S. H. Abbassi, I. M. Qureshi, B. R. Alyaei, H. Abbasi, and K. Sultan, "An efficient spectrum sensing mechanism for CRVANETs," Journal of Basic and Applied Scientific Research, vol. 3, no. 12, pp. 365-378, 2013.

[5] M. Di Felice, R. Doost-Mohammady, K. R. Chowdhury, and L. Bononi, "Smart radios for smart vehicles: cognitive vehicular 
networks," IEEE Vehicular Technology Magazine, vol. 7, no. 2, pp. 26-33, 2012.

[6] B. Karp and H. T. Kung, "GPSR: greedy perimeter stateless routing for wireless networks," in Proceedings of the ACM/IEEE International Conference on Mobile Computing and Networking (MobiCom '00), Boston, Mass, USA, August 2000.

[7] J. Zhao and G. Cao, "VADD: vehicle-assisted data delivery in vehicular ad hoc networks," IEEE Transactions on Vehicular Technology, vol. 57, no. 3, pp. 1910-1922, 2008.

[8] P.-C. Cheng, K. C. Lee, M. Gerla, and J. Härri, “GeoDTN+Nav: geographic DTN routing with navigator prediction for urban vehicular environments," Mobile Networks and Applications, vol. 15, no. 1, pp. 61-82, 2010.

[9] A. J. Ghandour, K. Fawaz, and H. Artail, "Data delivery guarantees in congested vehicular ad hoc networks using cognitive networks," in Proceedings of the 7th International IEEE Wireless Communications and Mobile Computing Conference (IWCMC '11), pp. 871-876, Istanbul, Turkey, July 2011.

[10] F. Riaz, I. Shafi, S. F. Hasan, W. Younas, and Y. Mehmood, "Vehicle-to-vehicle communication enhanced by cognitve approach and multi-radio technologies," in Proceedings of the International Conference on Emerging Technologies (ICET '12), pp. 131-136, Islamabad, Pakistan, October 2012.

[11] W. Kim, S. Y. Oh, M. Gerla, and K. C. Lee, "CoRoute: a new cognitive anypath vehicular routing protocol," in Proceedings of the 7th International Wireless Communications and Mobile Computing Conference (IWCMC '11), pp. 766-771, IEEE, Istanbul, Turkey, July 2011.

[12] J. Liu, P. Ren, S. Xue, and H. Chen, "Expected path duration maximized routing algorithm in CR-VANETs," in Proceedings of the 1st IEEE International Conference on Communications in China: Wireless Networking and Applications (ICCC '12), pp. 659-663, Beijing, China, August 2012.

[13] J. Kim and M. Krunz, "Spectrum-aware beaconless geographical routing protocol for cognitive radio enabled vehicular networks," Mobile Networks and Applications, vol. 18, no. 6, pp. 854-866, 2013.

[14] Y. Zang, L. Stibor, G. Orfanos, S. Guo, and H.-J. Reumerman, "An error model for inter-vehicle communications in highway scenarios at 5.9GHz," in Proceedings of the 2nd ACM International Workshop on Performance Evaluation of Wireless Ad Hoc, Sensor, and Ubiquitous Networks (PE-WASUN '05), pp. 49-56, ACM, Montreal, Canada, October 2005.

[15] T. Abbas, K. Sjöberg, J. Karedal, and F. Tufvesson, "A measurement based shadow fading model for vehicle-to-vehicle network simulations," International Journal of Antennas and Propagation, vol. 2015, Article ID 190607, 12 pages, 2015.

[16] J. Turkka and M. Renfors, "Path loss measurements for a nonline-of-sight mobile-to-mobile environment," in Proceedings of the 8th International Conference on ITS Telecommunications (ITST '08), pp. 274-278, IEEE, Phuket, Thailand, October 2008.

[17] IEEE, "IEEE trial-use standard for wireless access in vehicular environments (WAVE)-multi-channel operation," IEEE Standard 1609.4-2006, 2006.

[18] M. D. Felice, K. R. Chowdhury, and L. Bononi, "Analyzing the potential of cooperative cognitive radio technology on intervehicle communication," in Proceedings of the IFIP Wireless Days (WD '10), pp. 1-6, Venice, Italy, October 2010.

[19] A. S. B. Kozal, M. Merabti, and F. Bouhafs, "An improved energy detection scheme for cognitive radio networks in low SNR region," in Proceedings of the 17th IEEE Symposium on
Computers and Communication (ISCC '12), pp. 684-689, IEEE, Cappadocia, Turkey, July 2012.

[20] S. K. Dhurandher, M. S. Obaidat, D. Bhardwaj, and A. Garg, "GROOV: a geographic routing over VANETs and its performance evaluation," in Proceedings of the IEEE Global Communications Conference (GLOBECOM '12), pp. 1670-1675, Anaheim, Calif, USA, December 2012.

[21] M. Kasemann, H. Fubler, H. Hartenstein, and M. Mauve, "A reactive location service for mobile ad hoc networks," Tech. Rep. TR-02-014, Department of Computer Science, University of Mannheim, Mannheim, Germany, 2002.

[22] T. K. Vu and S. Kwon, "Mobility-assisted on-demand routing algorithm for MANETs in the presence of location errors," The Scientific World Journal, vol. 2014, Article ID 790103, 11 pages, 2014.

[23] H. Feng, C. Liu, Y. Shu, and O. W. W. Yang, "Location prediction of vehicles in VANETs using a Kalman filter," Wireless Personal Communications, vol. 80, no. 2, pp. 543-559, 2015.

[24] G. Welch and G. Bishop, An Introduction to the Kalman Filter, Department of Computer Science, University of North Carolina at Chapel Hill, Chapel Hill, NC, USA, 2001. 


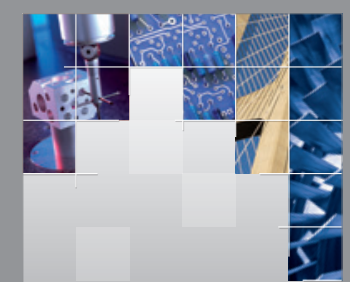

\section{Enfincering}
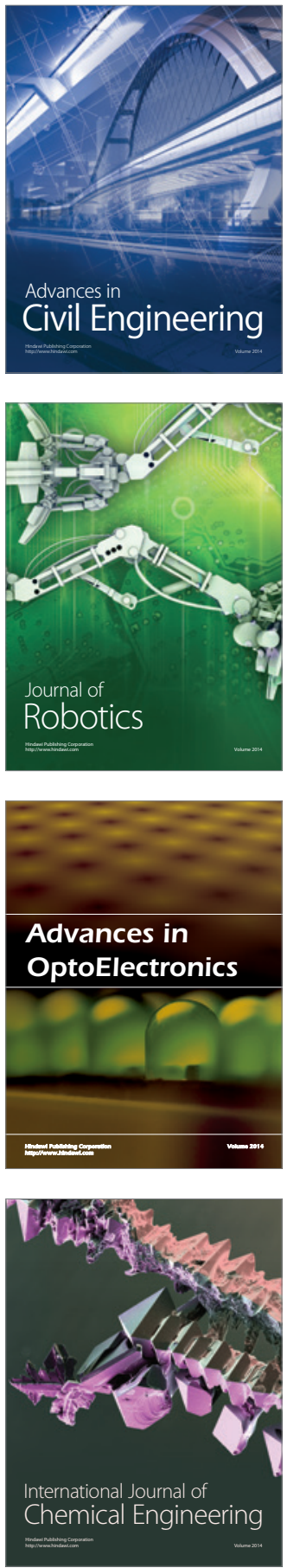

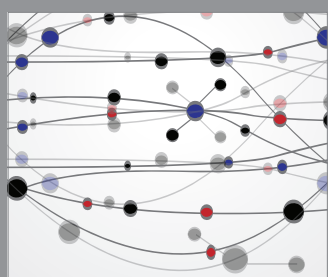

The Scientific World Journal

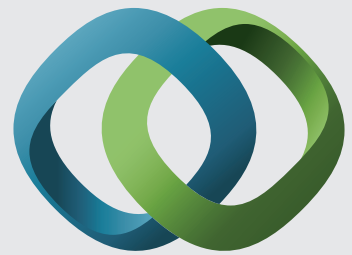

\section{Hindawi}

Submit your manuscripts at

http://www.hindawi.com
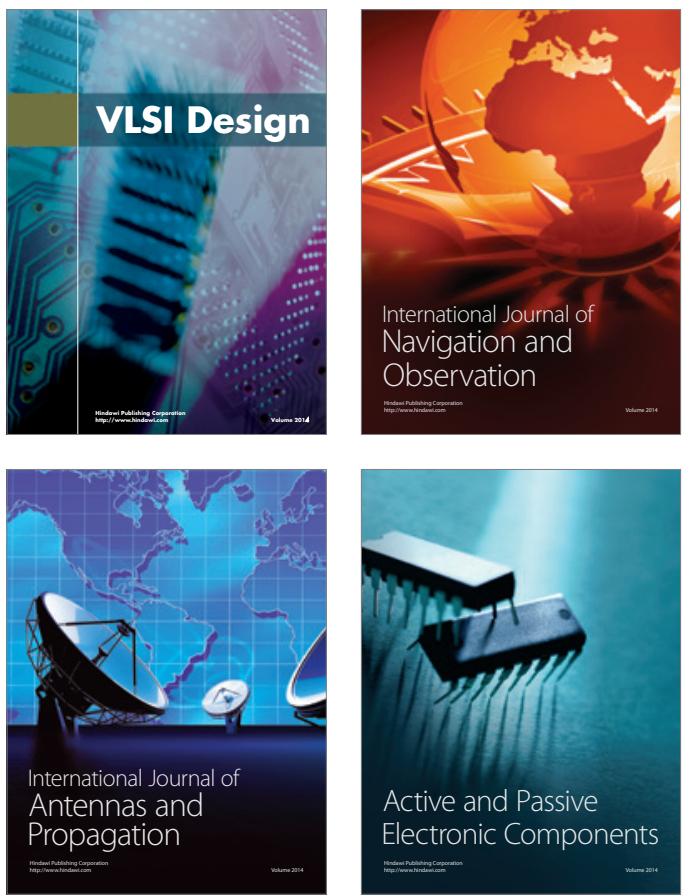
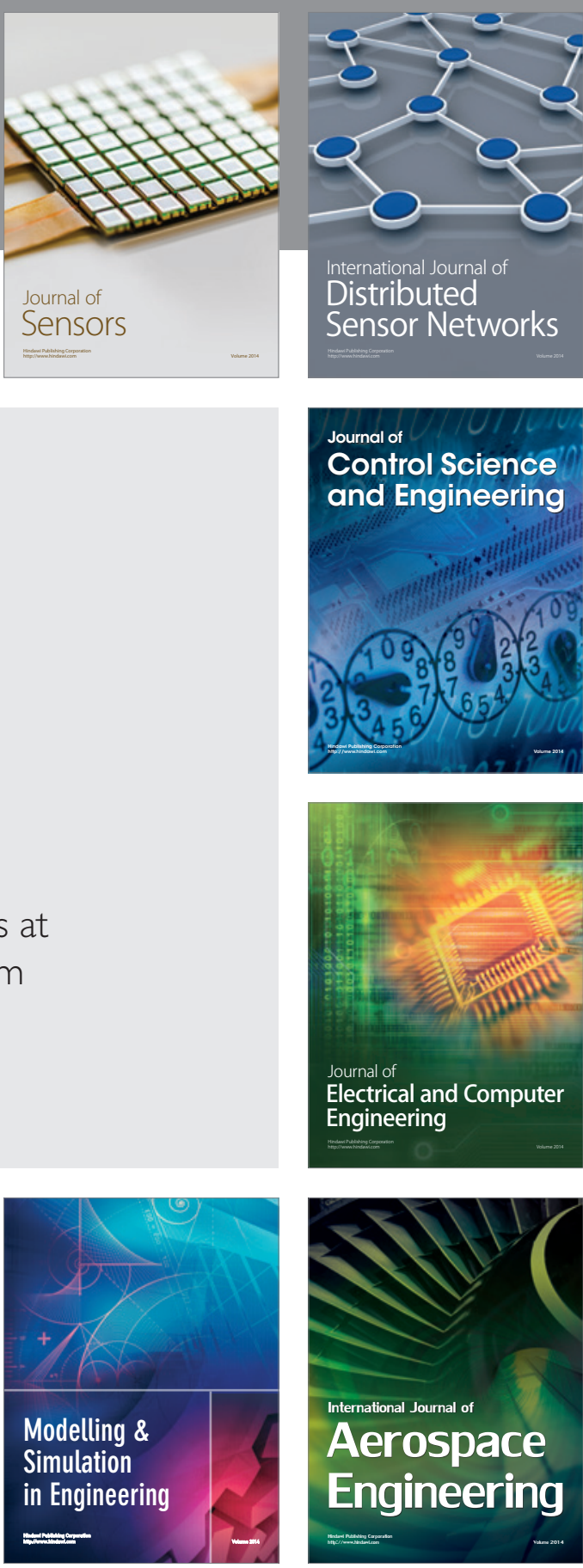

International Journal of

Distributed

Sensor Networks

Journal of

Control Science

and Engineering
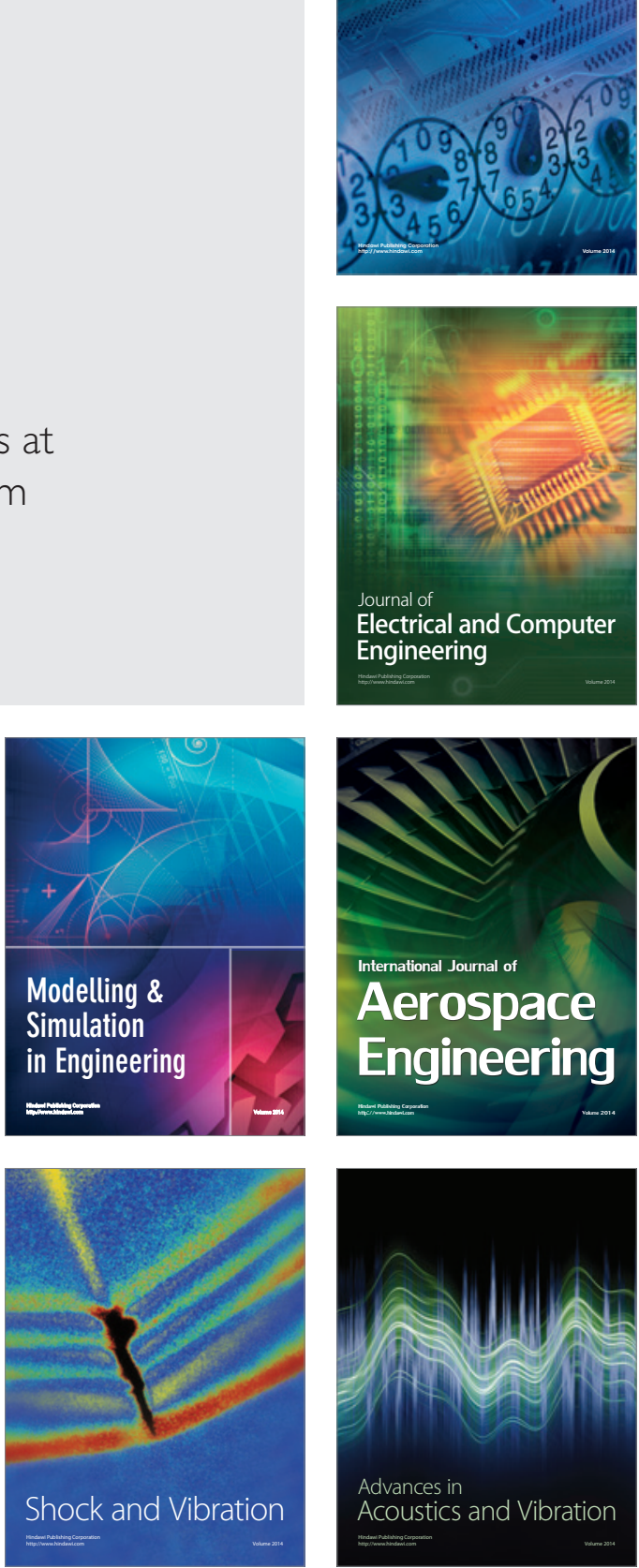